\title{
MicroRNA profiling associated with muscle growth in modern broilers compared to an unselected chicken breed
}

Bhuwan Khatri', Dongwon Seo ${ }^{1}$, Stephanie Shouse', Jeong Hoon Pan², Nicholas J. Hudson ${ }^{3}$, Jae Kyeom Kim², Walter Bottje ${ }^{1}$ and Byungwhi C. Kong ${ }^{1 *}$

\begin{abstract}
Background: Genetically selected modern broiler chickens have acquired outstanding production efficiency through rapid growth and improved feed efficiency compared to unselected chicken breeds. Recently, we analyzed the transcriptome of breast muscle tissues obtained from modern pedigree male (PeM) broilers (rapid growth and higher efficiency) and foundational Barred Plymouth Rock (BPR) chickens (slow growth and poorer efficiency). This study was designed to investigate microRNAs that play role in rapid growth of the breast muscles in modern broiler chickens.

Results: In this study, differential abundance of microRNA (miRNA) was analyzed in breast muscle of PeM and BPR chickens and the results were integrated with differentially expressed (DE) mRNA in the same tissues. A total of 994 miRNA were identified in PeM and BPR chicken lines from the initial analysis of small RNA sequencing data. After filtering and statistical analyses, the results showed miR-2131-5p, miR-221-5p, miR-126-3p, miR-146b-5p, miR-10a-5p, let-7b, miR-125b-5p, and miR-146c-5p up-regulated whereas miR-206 down-regulated in PeM compared to BPR breast muscle. Based on inhibitory regulations of miRNAs on the mRNA abundance, our computational analysis using miRDB, an online software, predicated that 118 down-regulated mRNAs may be targeted by the up-regulated miRNAs, while 35 up-regulated mRNAs appear to be due to a down-regulated miRNA (i.e., miR-206). Functional network analyses of target genes of DE miRNAs showed their involvement in calcium signaling, axonal guidance signaling, and NRF2-mediated oxidative stress response pathways suggesting their involvement in breast muscle growth in chickens.
\end{abstract}

Conclusion: From the integrated analyses of differentially expressed miRNA-mRNA data, we were able to identify breast muscle specific miRNAs and their target genes whose concerted actions can contribute to rapid growth and higher feed efficiency in modern broiler chickens. This study provides foundation data for elucidating molecular mechanisms that govern muscle growth in chickens.

Keywords: miRNA, Pedigree male broiler, Barred Plymouth Rock, Production efficiency, Differential expression

\section{Background}

Genetically selected modern broiler chickens are characterized by rapid growth and improved feed efficiency compared to unselected counterparts. These traits are beneficial to meet the global protein needs for an ever increasing human population $[1,2]$. Understanding the

\footnotetext{
*Correspondence: bkong@uark.edu

${ }^{1}$ Department of Poultry Science, Center of Excellence for Poultry Science,

University of Arkansas, Fayetteville, AR 72701, USA

Full list of author information is available at the end of the article
}

mechanism behind rapid muscle growth and high feed efficiency in chickens will help in maintaining sustainable protein source through improved animal production system.

MicroRNAs are short 18-24 nucleotides long non-coding regulatory RNAs that target mRNAs for cleavage, deadenylation or translational inhibition of gene expression at post-transcriptional level $[3,4]$. In recent years, many studies have shown the vital roles of miRNAs in various aspects of biological phenomenon associated with

(c) The Author(s). 2018 Open Access This article is distributed under the terms of the Creative Commons Attribution 4.0 International License (http://creativecommons.org/licenses/by/4.0/), which permits unrestricted use, distribution, and 
growth and development [5-8]. Recently, a total of 921 miRNAs were identified from breast muscles of fast and slow growing broilers [9]. Of note, let-7b was experimentally validated through genetic analyses in chickens to affect signaling pathways regulating skeletal muscle growth [10]. The miR-1 was shown to promote myogenesis by targeting histone deacetylase (HDAC) 4, a transcriptional repressor protein of muscle gene expression. The miR-133 was proven to enhance myoblast proliferation by repressing serum response factor (SRF) [11]. The miR-26a was reported to accelerate the process of myogenesis through induction of creatine kinase and up-regulation of myoD and myogenin [12]. All these reports suggest that miRNAs are important regulators of muscle growth and development in vertebrate animals.

Extensive genetic selection has led to rapid growth rate and large muscle mass in modern broilers compared to unselected chicken breeds [13]. In a previous study, we identified differentially expressed genes associated with breast muscle myogenesis in pedigree male (PeM) broilers (rapidly growing, higher efficiency, and large muscle mass) compared with Barred Plymouth Rock (BPR) chickens (slowly growing, poorer efficiency, and small muscle mass) [2]. This transcriptomic analysis indicated that rapid growth and large muscle mass shown in modern broilers may be due to altered mitochondrial functions, growth signaling pathways, oxidative stress pathway, and/or hormone receptor pathways. To elucidate regulatory roles of miRNAs on muscle growth and production efficiency, we profiled differentially expressed (DE) miRNAs using small RNA sequencing followed by prediction of potential target mRNAs; and eventually, miRNA profiling results were integrated with transcriptomic data of DE genes obtained by previous mRNA sequencing study [2].

\section{Methods}

\section{Ethics statement}

The present study was conducted in accordance with the recommendations in the guide for the care and use of laboratory animals of the National Institutes of Health. All procedures for animal care complied with the University of Arkansas Institutional Animal Care and Use Committee (IACUC): Protocol \#14012.

\section{Samples}

This study was conducted with modern pedigree male (PeM) broilers provided by Cobb Vantress, Inc. (Siloam Springs, AR) [14] and foundational Barred Plymouth Rock (BPR) chickens maintained at the Poultry Research Facilities of Arkansas Agricultural Experiment Station, University of Arkansas (Fayetteville, AR). Breast muscle tissues were obtained from PeM, highly selected for growth and feed efficiency [14, 15], and BPR as described elsewhere [2]. Briefly, immature PeM and BPR chickens ( $\leq 8$ weeks old, $n=6$ per breed) were killed by an overdose of sodium pentobarbital (i.v. injection) and breast muscle tissue was obtained and flash frozen in liquid nitrogen. Total RNAs were extracted from the muscle tissue using TRIzol reagent (Thermo-Fisher Scientific, Carlsbad, CA) following manufacturer's protocol. Extracted RNA samples were treated with DNase I (New England Biolabs Inc., Ipswich, MA) and purified again using TRIzol reagent. RNA quality was then assessed using Agilent 2200 TapeStation instrument (Santa Clara, CA). All RNA samples showed high enough quality and quantity (RNA Integrity Number; RIN > 5.5; data not shown), that were confirmed by sequencing core facility.

\section{MicroRNA sequencing and data analysis}

Library preparation for individual samples and sequencing were performed by the Research Technology Support Facility at Michigan State University (East Lansing, MI). Illumina TruSeq system $1 \times 50$ bp single end read method was used for miRNA sequencing. Quality of raw reads were determined using a FastQC tool kit [16] and adapters were trimmed using bbduk.sh command line of BBMap toolkit (https://sourceforge.n et/projects/bbmap/). The clean reads were then aligned to reference mature miRNA sequences of Gallus gallus obtained from miRBase (http://mirbase.org/) using the Arraystar program in Lasergene software package (DNAStar, Madison, WI) and read counts were normalized by reads per millions to stabilize the variance. Differential expression with normalized read counts was further analyzed using JMP Genomics 9 (SAS Institute Inc., Cary, NC). MicroRNAs with less than 5 average read counts in both comparison groups were not considered for further analysis. The $\mathrm{t}$-statistics was used to compare abundances between PeM and BPR, and miRNAs showing fold change $>1.2$ and $p$-value $<0.05$ were considered as DE.

\section{Hierarchical clustering}

DE miRNAs of PeM and BPR were subjected to hierarchical cluster analysis using JMP Genomics Program. A matrix with as many columns as birds (12) and as many rows as DE miRNA (9) were imported in which each cell contained $\log 2$ transformed fold change value for that miRNA and bird into JMP Genomics Program, normalizing on rows. After, hierarchical clustering on both rows and columns were applied followed by dendrogram image production.

\section{Target prediction of DE miRNA}

Online miRNA target prediction tool, miRDB (http:// www.mirdb.org/) was used to predict potential targets of 
DE miRNAs. The predicted targets of DE miRNA were then integrated with DE mRNA list obtained from same breast muscle tissue in our earlier study [2]. The mRNA showing opposite direction of expression to their corresponding miRNA were chosen as targets of DE miRNAs and used for subsequent bioinformatics analyses.

\section{Ingenuity pathway analysis}

Ingenuity Pathway Analysis (IPA; Qiagen, Valencia, CA; https://www.qiagenbioinformatics.com/) software was used for construction of interaction network between DE miRNA and their candidate targets. Since IPA is based on bioinformatics in humans, functionalities for DE miRNAs in the chicken datasets are principally based upon mammalian biological mechanisms. As investment in biomedical research biases the functional annotations towards human disease, we have attempted to draw plausible conclusions based on avian literature [2]. All target genes of DE miRNAs were subjected to IPA analysis for functional annotation and canonical pathways mapping among which all target genes were identified by IPA.

\section{Small RNA purification, CDNA synthesis and quantitative real time PCR (qPCR)}

Sixty micrograms of total RNA samples from 6 muscle samples each for PeM and BPR were used for small RNA enrichment and subsequent validation of miRNA sequencing results by qPCR. Small RNAs were enriched using mirVana miRNA isolation kit (Ambion, Carlsbad, CA) following manufacturer's instructions. Enriched small RNAs were polyadenylated using Poly(A) Polymerase (Ambion) and re-purified using QIAquick Nucleotide Removal Kit (Qiagen). The polyadenylated small RNAs were then ligated with RNA oligonucleotide adapter (Table 1), treated with RNaseOUT and reverse transcribed to cDNA using adapter primer and SuperScript III reverse transcriptase (Thermo-Fisher Scientific). The cDNA samples were diluted to 1:10 ratio and a portion $(2 \mu \mathrm{l})$ of $\mathrm{cDNA}$ was used for qPCR reaction using ABI prism $7500 \mathrm{HT}$ system (Thermo-Fisher Scientific) with PowerUp SYBR Green Master Mix (Thermo-Fisher Scientific). Primers were synthesized by Integrated DNA Technologies (Coralville, IA), and are listed in Table 1. The qPCR condition was as follows: 1 cycle at $95{ }^{\circ} \mathrm{C}$ for $2 \mathrm{~min}, 40$ cycles at $95{ }^{\circ} \mathrm{C}$ for $30 \mathrm{~s}, 60{ }^{\circ} \mathrm{C}$ for $30 \mathrm{~s}$. The chicken $5 \mathrm{~S}$ ribosomal RNA was used as internal control. Dissociation curves were generated at the end of amplification process for validating data quality. All qPCR reactions were conducted three times and values of average cycle threshold $(\mathrm{Ct})$ were determined for each sample, and $2^{-\Delta \Delta \mathrm{Ct}}$ values for the comparison of PeM and BPR were used for relative quantification by fold-change and statistical significance.
Table 1 Primers used for qPCR. The first column indicates primer names and the second column shows their sequences

\begin{tabular}{|c|c|}
\hline Name & Sequence \\
\hline RTQ_primer & 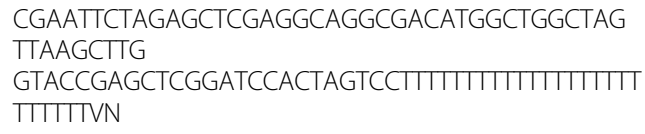 \\
\hline RTQ-UNIr & CGAATTCTAGAGCTCGAGGCAGG \\
\hline $\operatorname{miR}-146 c-5 p$ & TGAGAACTGAATTCCATGGACTG \\
\hline miR-146b-5p & TGAGAACTGAATTCCATAGGCG \\
\hline miR-10a-5p & TACCCTGTAGATCCGAATTTGT \\
\hline miR-2131-5p & CTGTTACTGTTCTTCTGATGG \\
\hline miR-221-5p & AACCTGGCATACAATGTAGATTTCTGT \\
\hline miR-10b-5p & TACCCTGTAGAACCGAATTTGT \\
\hline let-7b & TGAGGTAGTAGGTTGTGTGGTT \\
\hline miR-125b-5p & TCCCTGAGACCCTAACTTGTGA \\
\hline miR-206 & TGGAATGTAAGGAAGTGTGTGG \\
\hline 5S_rRNA-F1 & AAGCCTACAGCACCCGGTAT \\
\hline
\end{tabular}

\section{Results}

MicroRNA profiling in PeM and BPR chickens by miRNA sequencing

MicroRNA Sequencing of 12 samples yielded 33,727,148 and 51,310,328 raw sequence reads from PeM and BPR samples, respectively. After adapter trimming, $17,751,585$ and 22,922,027 clean reads remained in PeM and BPR, respectively (data not shown). After alignment of clean reads to chicken reference mature miRNA collections, a total of 994 mature miRNAs were identified in both PeM and BPR. Rarely expressed mature miRNAs (i.e., raw read count $<5$ ) were filtered out, resulting in 38 miRNAs remained as meaningfully expressed and were used for subsequent analyses (Additional file 1).

\section{Differentially expressed miRNAs in PeM compared to BPR}

Nine DE miRNAs showing $p$-value $<0.05$ and fold change $>1.2$ were identified in PeM compared with BPR (Table 2). Among 9 DE miRNAs, 8 miRNAs including miR-2131-5p, miR-221-5p, miR-126-3p, miR-146b-5p, miR-10a-5p, let-7b, miR-125b-5p, and miR-146c-5p were up-regulated while miR-206 was down-regulated in PeM compared to BPR breast muscle (Table 2). All DE miRNAs were validated using qPCR (Table 2). Our qPCR results indicated that expression patterns of 8 out of 9 miRNAs were in good agreement with miRNA sequencing data in terms of their direction and magnitude of change. One (miR-126-3p) out of 9 miRNAs did not match with miRNA sequencing which may be due to different approaches for data normalization. Additionally, hierarchical clustering showed clear discrimination of 12 birds into correct group of origin (Fig. 1). 
Table 2 Comparison of fold change between miRNAseq and qPCR in breast muscle tissue of PeM compared to BPR broilers

\begin{tabular}{|c|c|c|c|c|}
\hline \multirow[t]{2}{*}{ miRNA } & \multirow[t]{2}{*}{ Sequence } & \multicolumn{2}{|c|}{ miRNAseq } & \multirow{2}{*}{$\begin{array}{l}\mathrm{qPCR} \\
\mathrm{FC} \pm \mathrm{SEM}^{\mathrm{b}}\end{array}$} \\
\hline & & $\overline{\mathrm{FC}^{\mathrm{a}}}$ & $p$-value & \\
\hline miR-2131-5p & AUGCAGAAGUGCACGGAAACAGCU & 2.62 & $5.71 \mathrm{E}-05$ & $1.45 \pm 0.24$ \\
\hline miR-221-5p & AACCUGGCAUACAAUGUAGAUUUCUGU & 2.42 & 0.001 & $1.43 \pm 0.31$ \\
\hline $\operatorname{miR}-126-3 p^{c}$ & UCGUACCGUGAGUAAUAAUGCGC & 1.49 & 0.002 & $-0.87 \pm 0.15^{c}$ \\
\hline miR-146b-5p & UGAGAACUGAAUUCCAUAGGCG & 2.44 & 0.005 & $1.88 \pm 0.30$ \\
\hline miR-10a-5p & UACCCUGUAGAUCCGAAUUUGU & 1.96 & 0.005 & $1.17 \pm 0.10$ \\
\hline miR-206 & UGGAAUGUAAGGAAGUGUGUGG & -0.72 & 0.017 & $-0.62 \pm 0.11$ \\
\hline let-7b & UGAGGUAGUAGGUUGUGUGGUU & 1.20 & 0.027 & $1.20 \pm 0.16$ \\
\hline miR-125b-5p & UCCCUGAGACCCUAACUUGUGA & 1.51 & 0.028 & $1.17 \pm 0.09$ \\
\hline miR-146c-5p & UGAGAACUGAAUUCCAUGGACUG & 1.42 & 0.049 & $1.31 \pm 0.13$ \\
\hline
\end{tabular}

${ }^{a}$ Values denote linear fold changes obtained from miRNAseq analyses

${ }^{b}$ Values denote linear fold changes plus/minus standard error of the mean (SEM) from qPCR

Indicate inconsistent fold change between RNAseq and qPCR

\section{Target prediction and network analysis}

In our recent study, DE mRNAs were identified by mRNA sequencing analysis in the same breast muscle tissues of PeM and BPR [2]. To investigate potential interactions between miRNA and mRNA expression, miRDB (http://mirdb.org), an online tool for miRNA target prediction and functional annotations was used to predict target genes of validated, DE miRNAs. A total of 2194 genes (mRNAs) were predicted as potential targets for 8 qPCR validated DE miRNAs (except miR-126-3p which showed inconsistent fold change values between miRNAseq and qPCR) (data not shown). Target mRNAs for DE miRNA were integrated with our DE mRNA dataset (retrieved from Kong et al., 2017). Expressions showing opposite direction to corresponding miRNA (e.g., down-regulated transcripts that are targets of up-regulated miRNA in PeM) were chosen for further pathway analysis using the IPA. According to the miRNA-mRNA interaction criteria, 153 candidate target genes for 8 miRNAs were identified (Additional file 2) including 118 down-regulated transcripts potentially targeted by 7 up-regulated miRNAs (miR-2131-5p, miR221-5p, miR-146b-5p, miR-10a-5p, let-7b, miR-125b-5p, and miR-146c-5p). Similarly, it was predicted that 35 up-regulated transcripts might be modulated by downregulated miRNA (miR-206) in PeM muscle samples.

As results of pathway analyses with DE miRNA and their target genes, the top biological functions of target genes were identified by the IPA using its features, "Top Canonical Pathways" and "Physiological System Development and Function" (Table 3). The most relevant biological functions of DE miRNA and their target DE mRNA in skeletal muscle included axonal guidance signaling, glycine degradation, calcium

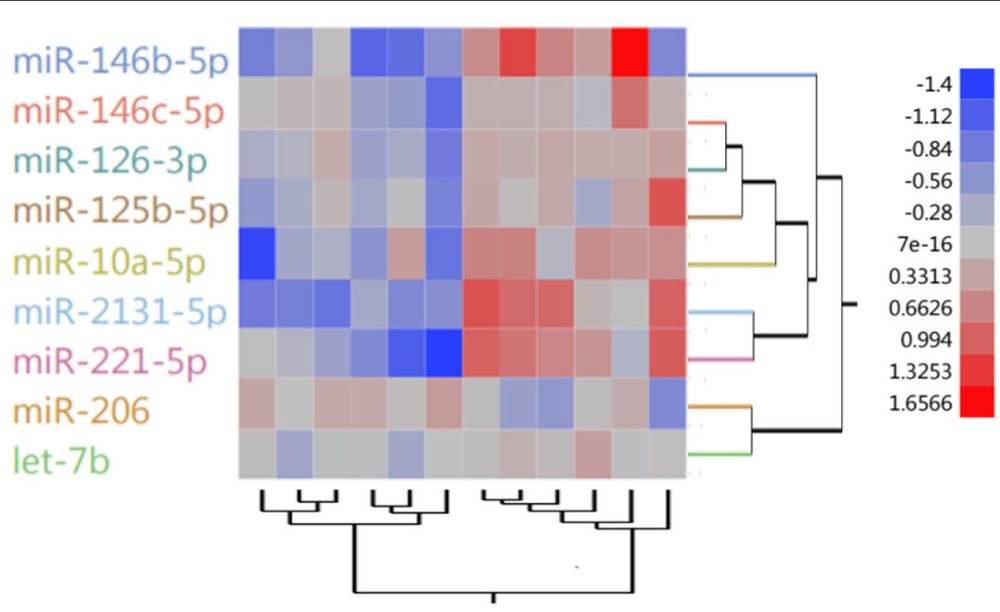

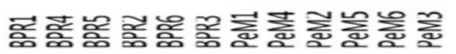

Fig. 1 Hierarchically clustered heat map of 9 DE miRNA. Red and blue represent up and down-regulated expression in PeM respectively. Color density indicated level of fold change 
Table 3 Top biological functions of target genes between PeM and BPR as presented by IPA

\begin{tabular}{ll}
\hline Top Canonical Pathways & $p$-value \\
\hline Axonal Guidance Signaling & 0.00991 \\
Glycine Degradation (Creatine Biosynthesis) & 0.0131 \\
Calcium Signaling & 0.0303 \\
Serine Biosynthesis & 0.0325 \\
NRF2-mediated oxidative stress response & 0.0361 \\
Zymosterol Biosynthesis & 0.0389 \\
Physiological System Development & $p$-value range \\
and Function & \\
Endocrine System Development & $1.96 \mathrm{E}-02-2.56 \mathrm{E}-04$ \\
and Function & \\
Embryonic Development & $2.86 \mathrm{E}-02-4.33 \mathrm{E}-04$ \\
Organismal Development & $2.92 \mathrm{E}-02-4.33 \mathrm{E}-04$ \\
Skeletal and Muscular System & $2.61 \mathrm{E}-02-6.36 \mathrm{E}-04$ \\
Developmental and Function & \\
Tissue Development & $2.86 \mathrm{E}-02-8.86 \mathrm{E}-04$ \\
\hline
\end{tabular}

signaling, serine biosynthesis, zymosterol biosynthesis, endocrine system development and function, embryonic development, organismal development, skeletal and muscular system development and function, and tissue organismal development.

\section{Discussion}

In this study, an extensive set of miRNAs was identified by small RNA sequencing and their potential roles in muscle growth and feed efficiency were determined in PeM and BPR chickens. Among a total of 994 mature miRNAs identified (data not shown), the 38 mature miRNAs were abundantly present in breast muscle of PeM and BPR birds (Table 4). Of those, miR-1a-3p was the most abundant with average read per million reads (RPM) of $334,936.0$ and $337,938.6$ in BPR and PeM chickens, respectively (Table 4 ). A study conducted in mice showed the role of the elevated miR-1a-3p in suppressing multiple factors required for the phosphorylation of the c-Jun N-terminal kinases/Mitogen activated protein kinase (JNK/MAPK) pathway and thereby promoting the expression of transcriptional factor MyoD [17], a myogenic regulatory factor required for skeletal muscle development [18]. However, the role of miR-1a$3 p$ in muscle growth in chickens has not been studied. The other miRNA, miR-133c-3p, that was abundantly expressed in chicken breast muscle [19], enhances skeletal muscle proliferation and differentiation by repressing SRF [11]. Similarly, miR-21 and miR-146c have been recently identified as miRNAs upregulated in chicken interdigital regression and associated with inflammation, cell senescence, and programed cell death

Table 4 Abundant mature miRNAs present in breast muscle of PeM and BPR chickens

\begin{tabular}{|c|c|c|c|c|c|c|c|}
\hline \multirow[b]{2}{*}{ miRNA } & \multicolumn{3}{|l|}{ *RPM } & \multirow[b]{2}{*}{ miRNA } & \multicolumn{3}{|l|}{ *RPM } \\
\hline & Ave. BPR & Ave. PeM & ${ }^{* *}$ Mean & & Ave. BPR & Ave. PeM & ${ }^{* *}$ Mean \\
\hline gga-miR-1a-3p & $334,936.0$ & $337,938.6$ & $336,437.3$ & gga-miR-133a-5p & 6298.4 & 6064.0 & 6181.2 \\
\hline gga-miR-30c-5p & $186,869.2$ & $178,733.7$ & $182,801.4$ & gga-miR-10b-5p & 5047.2 & 8048.4 & 6547.8 \\
\hline gga-miR-126-3p & $49,349.8$ & $71,550.1$ & $60,450.0$ & gga-miR-24-3p & 5001.3 & 4388.1 & 4694.7 \\
\hline gga-miR-17-5p & $58,039.9$ & $53,308.0$ & $55,673.9$ & gga-miR-16-5p & 4105.2 & 5268.3 & 4686.8 \\
\hline gga-let-7b & $26,643.5$ & $31,809.5$ & $29,226.5$ & gga-miR-301b-3p & 4767.5 & 4666.8 & 4717.1 \\
\hline gga-miR-181a-5p & $29,530.8$ & $26,834.5$ & $28,182.7$ & gga-miR-221-5p & 2740.1 & 6499.7 & 4619.9 \\
\hline gga-miR-206 & $31,760.2$ & $23,406.7$ & $27,583.5$ & gga-miR-21-5p & 3496.3 & 3803.9 & 3650.1 \\
\hline gga-let-7f-5p & $19,774.2$ & $16,839.1$ & $18,306.6$ & gga-miR-20a-5p & 3395.8 & 3528.7 & 3462.3 \\
\hline gga-let-7c-5p & $15,467.4$ & $16,304.0$ & $15,885.7$ & gga-miR-451 & 4919.9 & 2846.0 & 3883.0 \\
\hline gga-miR-30d & $16,441.3$ & $15,214.6$ & $15,827.9$ & gga-miR-10a-5p & 2409.5 & 4459.7 & 3434.6 \\
\hline gga-miR-146c-5p & $12,167.9$ & $17,007.6$ & $14,587.8$ & gga-miR-221-3p & 2993.7 & 3519.9 & 3256.8 \\
\hline gga-miR-222a & $10,032.7$ & $12,212.6$ & $11,122.6$ & gga-let-7 g-5p & 3044.1 & 3207.3 & 3125.7 \\
\hline gga-miR-133c-3p & 9874.8 & $11,030.0$ & $10,452.4$ & gga-miR-103-3p & 2819.7 & 2995.4 & 2907.6 \\
\hline gga-miR-30a-5p & 9682.9 & $10,924.1$ & $10,303.5$ & gga-miR-22-3p & 1988.3 & 2714.4 & 2351.3 \\
\hline gga-miR-193a-3p & $11,370.2$ & $10,310.7$ & $10,840.4$ & gga-miR-2954 & 1977.5 & 1520.8 & 1749.2 \\
\hline gga-miR-125b-5p & 8184.5 & $12,684.1$ & $10,434.3$ & gga-miR-1454 & 1545.4 & 1526.3 & 1535.9 \\
\hline gga-miR-26a-5p & 8965.0 & 7300.4 & 8132.7 & gga-miR-2131-5p & 927.1 & 2505.3 & 1716.2 \\
\hline gga-miR-199-5p & 8310.5 & 7656.8 & 7983.6 & gga-miR-128-3p & 1276.4 & 1466.2 & 1371.3 \\
\hline gga-miR-148a-3p & 6703.5 & 6804.6 & 6754.1 & gga-miR-146b-5p & 713.6 & 1894.0 & 1303.8 \\
\hline
\end{tabular}


[20]. Additionally, a previous study reported upregulated expression of miR-21 in breast muscle of low body weight chickens [9]. Other studies in chicken and rats have reported the inhibitory effect of miR-21 in proliferation of pre-adipocytes and renal tubular epithelial cells, respectively $[21,22]$. In addition, miR-22-3p, miR-30a-5p, miR-30d, miR-10b, miR-148a, miR-146c-5p and miR-199 were also known as abundantly present in breast muscles [9]. Altogether, evidences in literature suggest that abundant miRNAs identified in our samples may play role in enhanced growth and development of breast muscle of modern broilers.

Of eight DE miRNAs validated with qPCR, expressions of miR-146b-5p, miR-125b-5p, miR-2131-5p, let-7b, miR-221-5p miR-146c-5p, miR-126-3p, and miR-10a-5p were higher in PeM muscle while the miR-206 showed lowered expression in PeM. All DE miRNAs have been shown to be involved in muscle development in various animal species [5, 7, 9, 23-25]. MiR-206 is specifically expressed in skeletal muscle and functions in muscle differentiation and cell proliferation in chickens and human [26-28]. Let-7b has been reported to be abundantly expressed in breast and skeletal muscle in chickens and to play roles in growth regulation via targeting growth hormone receptor [29]. MiR-10a is a well characterized
miRNA and is known to implicate with muscle development and myogenesis regulation in various animals [7, 23, 24, 30]. MiR-146b is a known regulator of skeletal myoblast differentiation in vitro and muscle regeneration in mice [25]. MiR-126 mediates vascular integrity and angiogenesis. It also elicits direct effects on regulation of skeletal muscle growth and activation of insulin like growth factor 1(IGF-1) [31, 32]. MiR-125b is known to regulate calcification of vascular smooth muscle cells. It targets IGF in both regenerating muscles and myoblasts [33]. There is evidence of expression of miR-221 controlled by Ras-MAPK pathway. It is involved in vascular smooth muscle proliferation and plays a role in progression from myoblasts to myocytes developing into fully differentiated phenotype $[10,34]$. Therefore, all DE miRNAs identified in this study seem to be closely related with muscle growth and development in chickens to their mammalian counterparts.

To understand cellular and physiological mechanisms in chickens muscle development, DE miRNA and their target DE mRNA were subjected to in silico pathway analysis using the IPA. The network analysis of DE miRNA-DE mRNA pairs (showing opposite expression patterns between miRNA and DE mRNA) were shown to be interlinked with P38 MAPK, ERK1/2, PI3K, and








Fig. 3 Pathway of calcium signaling predicted by IPA. Color symbols were indicated in the legend of Fig. 2

insulin-signaling pathways (Fig. 2). Further, functions associated with these genes include embryonic development, organ development, organismal development, and molecular transport. In the network, the up-regulation of annexin A2 (ANXA2), tropomyosin 3 (TPM3) and eukaryotic translation initiation factor 2 alpha kinase 3 (EIF2AK3) genes seemed to be directly regulated by down-regulation of miR-206 and their close association with P38 MAPK, ERK1/2, PI3K and insulin signaling cascades. ANXA2, lipocortin II or p36, which is a $36-\mathrm{kDa}$ $\mathrm{Ca}^{2+}$-dependent protein of the annexin superfamily plays regulatory functions in proliferation, migration and cytoskeletal formation in muscle cells [35, 36]. TPM3 binds to actin filaments in muscle cells. In association with troponin complex, TPM3 has central role in controlling contraction of striated muscle in vertebrates [37]. EIF2AK3, a metabolic stress sensing protein kinase, phosphorylates eukaryotic translation initiation factor 2 and is also involved in controlling mitochondrial morphology and function $[38,39]$. Therefore, it is possible that the interaction between miR-206 and its target genes may reflect the rapid myogenesis shown in breast muscle of PeM chickens. Interestingly, the down-regulated target genes of miR-146, miR-10a-5p, miR-125b-5p, miR-2131-5p and let-7b are also assigned to these signaling pathways.

From the IPA canonical pathway analysis, we found that the targeted DE mRNAs were associated with calcium signaling ( $p$-value 3.03E-02; Fig. 3), axonal guidance signaling (p-value 9.91E-03; Fig. 4), and NRF2-mediated oxidative stress response (p-value 3.61E-02; Fig. 5) pathways. The target genes involved in calcium signaling pathway include: HDAC11 gene (target of miR-10a-5p); a member of RAS oncogene family (RAP1B) and nuclear factor of activated T-cells 1 (NFATC1) genes (targets of miR-2131-5p); and TPM3 and bone morphogenetic protein 6 genes (BMP6) (targets of miR-206). It is well established that the homeostasis of intracellular calcium level is important for muscle growth and development. The increased intracellular level of $\mathrm{Ca}^{2+}$ can occur due to both poor $\mathrm{Ca}^{2+}$-ATPase activity and disturbance of sarcolemma integrity which results in

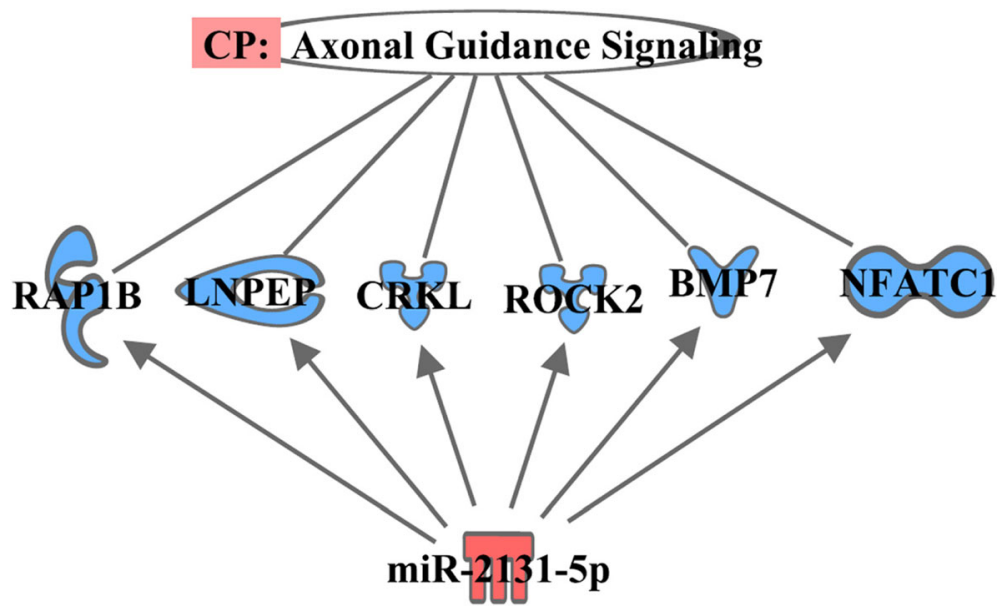

Fig. 4 Pathway of axonal guidance. Color symbols were indicated in the legend of Fig. 2 


\section{CP: NRF2-mediated Oxidative Stress Response}

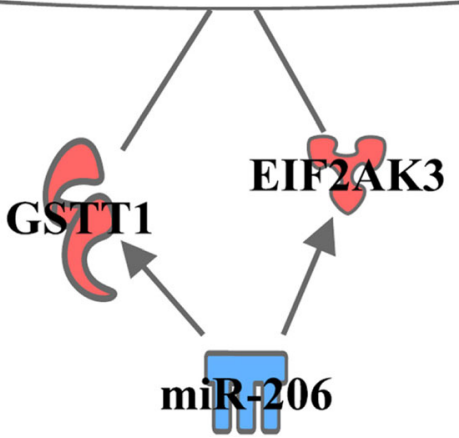

Fig. 5 Pathway of NRF2-mediated oxidative stress response. Color symbols were indicated in the legend of Fig. 2

hypercontraction of myofibers and degeneration of muscle mass [40-43]; the IPA suggests that this pathway might have been modulated by miR-10a-5p and miR-2131-5p thereby leading to larger muscle mass observed in PeM compared to BPR chickens. We also found the involvement of target genes of miR-2131-5p in axonal guidance signaling pathway; the predicted target mRNAs of miR-2131-5p included RAP1B, leucyl and cystinyl aminopeptidase, CRK like proto-oncogene, adapter protein, Rho associated coiled-coil containing protein kinase 2, BMP7, and NFATC1. Interestingly, Mutryn et al. (2015) recently suggested possible roles of axonal guidance signaling pathway in the breast muscle myopathy in chickens [40]. In a different study, miR-2131-5p has been reported as a mature miRNA specific to the avian lineage with unknown functions [44]. Hence, it is postulated that DE miR-2131-5p can play roles in regulation of axonal guidance signaling pathway in breast muscle of chickens. Further investigations and validation works might be warranted with regards to the implications of calcium metabolism and axonal guidance signaling in PeM samples and responsible physiological roles of DE miRNAs therein.

Last, we identified EIF2AK3 and glutathione S-transferase theta 1 (GSTT1) genes (targets of miR-206) involved in NRF2-mediated oxidative stress response pathway. NRF2 is one of the main factors responding to both oxidative and xenobiotic stresses. It plays a critical role in neutralizing oxidative stress by activating the expression of antioxidants and detoxifying enzymes [45]. The activation of EIF2AK3 and GSTT1 genes is associated with decreased level of reactive oxygen species $[46,47]$. Previously, the augmentation of the NRF2mediated oxidative stress response pathway is observed in breast muscle of higher feed efficient and rapidly growing chickens [48]. Therefore, increased expression of EIF2AK3 and GSTT1 genes, as a result of down-regulation of miR-206, implied the activation of canonical NRF2-mediated response pathway for scavenging reactive oxygen species from breast muscle of rapidly growing PeM chickens.

\section{Conclusion}

Using miRNA sequencing, integrated analyses of miRNA-mRNA data and IPA, we were able to identify breast muscle specific miRNAs and their target genes whose concerted actions can contribute to rapid growth and higher feed efficiency in modern broiler chickens. We believe our comprehensive analysis enables us to better understand miRNA and their physiological roles in breast muscle growth in chickens. Future validation studies are warranted in regards to interactions between miRNA and target genes (e.g., in vitro transfection studies) to characterize functions of miRNAs and their specific targets in the context of rapid muscle growth and development.

\section{Additional files}

Additional file 1: Read Per Million values of abundantly expressed miRNAs for indivial samples. (XLSX $22 \mathrm{~kb}$ )

Additional file 2: Target mRNAs of differentially expressed and $\mathrm{qPCR}$ validated miRNAs in PeM and BPR comparison. (XLSX 20 kb)

\footnotetext{
Abbreviations

ANXA2: Annexin A2; ATP: Adenosine triphosphate; BMP7: Bone morphogenetic protein 7 gene; BPR: Barred Plymouth Rock; cDNA: Complementary DNA;

Ct: Cycle threshold; DE: Differentially expressed; EIF2AK3: Eukaryotic translation initiation factor 2 alpha kinase 3; ERK1/2: Extracellular signal-regulated kinase; GSTT1: Glutathione S-transferase theta 1; HDAC: Histone deacetylase; IACUC: Institutional Animal Care and Use Protocol; IGF-1: Insulin like growth factor 1; IPA: Ingenuity Pathway Analysis; JNK: c-Jun N-terminal kinases; MAPK: Mitogen activated protein kinase; miRNA: MicroRNA; mRNA: messenger RNA; NFATC1: Nuclear factor of activated T-cells 1; NRF2: Nuclear related factor 2; PeM: Pedigree Male; PI3K: Phosphoinositide 3-kinase; qPCR: quantitative reverse transcription polymerase chain reaction; RAP1B: RAS oncogene family; SRF: Serum response factor; TPM3: Tropomyosin 3
} 


\section{Acknowledgements}

We thank Arkansas High Performance Computing and Cell and Molecular Biology Graduate Program of University of Arkansas for supporting cluster computing resources and JMP Genomics 9, respectively.

\section{Funding}

This study was funded by USDA-NIFA (\#2013-01953), Arkansas Biosciences Institute (ABI: Little Rock, AR) and the Agricultural Experiment Station (Univ. of Arkansas, Fayetteville). The USDA-NIFA and ABI provided the costs of small RNA sequencing, IPA license and GPCR validation of DE miRNAs. The Agricultural Experiment Station provided chickens and graduate assistantship.

\section{Availability of data and materials}

Datasets supporting the results of this article are also included in the Additional file. All the raw and normalized data are deposited in the Gene Expression Omnibus (GEO; accession number: GSE119528).

\section{Authors' contributions}

$\mathrm{BK}, \mathrm{BCK}, \mathrm{NH}, \mathrm{JK}$, and WB designed and performed the experiments, analyzed the data, bioinformatic analyses and wrote the manuscript. BK, BCK, DS, JP, and SS analyzed RNAseq data and analyzed GPCR assay. All authors read and approved the final manuscript.

\section{Ethics approval and consent to participate}

All procedures for animal care complied with the University of Arkansas Institutional Animal Care and Use Committee (IACUC): Protocol \#14012.

\section{Consent for publication}

Not applicable.

\section{Competing interests}

The authors declare that they have no competing interests.

\section{Publisher's Note}

Springer Nature remains neutral with regard to jurisdictional claims in published maps and institutional affiliations.

\section{Author details}

'Department of Poultry Science, Center of Excellence for Poultry Science, University of Arkansas, Fayetteville, AR 72701, USA. ${ }^{2}$ School of Human Environmental Sciences, University of Arkansas, Fayetteville, AR 72701, USA. ${ }^{3}$ School of Agriculture and Food Sciences, The University of Queensland, QLD4343, Gatton, Australia.

Received: 22 May 2018 Accepted: 7 September 2018

Published online: 17 September 2018

\section{References}

1. Kong B, Lassiter K, Piekarski-Welsher A, Dridi S, Reverter-Gomez A, Hudson $\mathrm{NJ}$, et al. Proteomics of Breast Muscle Tissue Associated with the Phenotypic Expression of Feed Efficiency within a Pedigree Male Broiler Line: I. Highlight on Mitochondria. PloS One. 2016;11(5):e0155679.

2. Kong BW, Hudson N, Seo D, Lee S, Khatri B, Lassiter K, et al. RNA sequencing for global gene expression associated with muscle growth in a single male modern broiler line compared to a foundational Barred Plymouth Rock chicken line. BMC Genomics. 2017;18(1):82. https://doi.org/ 10.1186/s12864-016-3471-y.

3. Bushati N, Cohen SM. microRNA functions. Annu Rev Cell Dev Biol. 2007;23:175-205.

4. Rana TM. Illuminating the silence: understanding the structure and function of small RNAs. Nat Rev Mol Cell Biol. 2007;8(1):23-36.

5. Anderson C, Catoe H, Werner R. MIR-206 regulates connexin43 expression during skeletal muscle development. Nucleic Acids Res. 2006;34(20):5863-71.

6. Gangaraju VK, Lin H. MicroRNAs: key regulators of stem cells. Nat Rev Mol Cell Biol. 2009;10(2):116-25.

7. Li T, Wu R, Zhang Y, Zhu D. A systematic analysis of the skeletal muscle miRNA transcriptome of chicken varieties with divergent skeletal muscle growth identifies novel miRNAs and differentially expressed miRNAs. BMC Genomics. 2011;12(1):186.
8. Wienholds E, Kloosterman WP, Miska E, Alvarez-Saavedra E, Berezikov E, de


Science. 2005;309(5732):310-1.

9. Ouyang $H$, He X, Li G, Xu H, Jia X, Nie Q, et al. Deep sequencing analysis of miRNA expression in breast muscle of fast-growing and slow-growing broilers. Int J Mol Sci. 2015;16(7):16242-62.

10. Liu X, Cheng Y, Zhang S, Lin Y, Yang J, Zhang C. A necessary role of miR221 and miR-222 in vascular smooth muscle cell proliferation and neointimal hyperplasia. Circ Res. 2009;104(4):476-87.

11. Chen J, Mandel EM, Thomson JM, Wu Q, Callis TE, Hammond SM, et al. The role of microRNA-1 and microRNA-133 in skeletal muscle proliferation and differentiation. Nat Genet. 2006;38(2):228-33.

12. Wong CF, Tellam RL. MicroRNA-26a targets the histone methyltransferase enhancer of Zeste homolog 2 during myogenesis. J Biol Chem. 2008; 283(15):9836-43.

13. Lopez G, De Lange K, Leeson S. Partitioning of retained energy in broilers and birds with intermediate growth rate. Poult Sci. 2007:86(10):2162-71.

14. Bottje W, lqbal M, Tang ZX, Cawthon D, Okimoto R, Wing T, et al. Association of mitochondrial function with feed efficiency within a single genetic line of male broilers. Poult Sci. 2002;81(4):546-55.

15. Kong BW, Song JJ, Lee JY, Hargis BM, Wing T, Lassiter K, et al. Gene expression in breast muscle associated with feed efficiency in a single male broiler line using a chicken $44 \mathrm{~K}$ oligo microarray. I. Top differentially expressed genes. Poult Sci. 2011;90(11):2535-47.

16. Andrews S. FastQC: a quality control tool for high throughput sequence data. 2010.

17. Xie S, Li J, Chen H, Tan Y, Liu S, Zhang Y, et al. Inhibition of the JNK/MAPK signaling pathway by myogenesis-associated miRNAs is required for skeletal muscle development. Cell Death Differ. Published Online. 2018. https://doi. org/10.1038/s41418-018-0063-1.

18. Bentzinger CF, Wang YX, Rudnicki MA. Building muscle: molecular regulation of myogenesis. Cold Spring Harb Perspect Biol. 2012:4(2):10.

19. Sweetman D, Goljanek K, Rathjen T, Oustanina S, Braun T, Dalmay T, et al. Specific requirements of MRFs for the expression of muscle specific microRNAs, miR-1, miR-206 and miR-133. Dev Biol. 2008;321(2):491-9.

20. Garcia-Riart B, Lorda-Diez Cl, Marin-Llera JC, Garcia-Porrero JA, Hurle JM, Montero JA. Interdigital tissue remodelling in the embryonic limb involves dynamic regulation of the miRNA profiles. J Anat. 2017:231(2): 275-86.

21. Wang W, Cheng M, Qiao S, Wang Y, Li H, Wang N. Gga-miR-21 inhibits chicken pre-adipocyte proliferation in part by down-regulating Kruppel-like factor 5. Poult Sci. 2016;96(1):200-10.

22. Lin L, Gan H, Zhang H, Tang W, Sun Y, Tang X, et al. MicroRNA-21 inhibits SMAD7 expression through a target sequence in the 3'untranslated region and inhibits proliferation of renal tubular epithelial cells. Mol Med Rep. 2014 10(2):707-12.

23. Hu R, Pan W, Fedulov AV, Jester W, Jones MR, Weiss ST, et al. MicroRNA-10a controls airway smooth muscle cell proliferation via direct targeting of the PI3 kinase pathway. FASEB J. 2014;28(5):2347-57.

24. Huang H, Xie C, Sun X, Ritchie RP, Zhang J, Chen YE. miR-10a contributes to retinoid acid-induced smooth muscle cell differentiation. J Biol Chem. 2010; 285(13):9383-9.

25. Khanna N, Ge Y, Chen J. MicroRNA-146b promotes myogenic differentiation and modulates multiple gene targets in muscle cells. PLoS One. 2014;9(6):e100657.

26. Jia X, Lin H, Abdalla BA, Nie Q. Characterization of miR-206 promoter and its association with birthweight in chicken. Int J Mol Sci. 2016;17(4):559.

27. Koutsoulidou A, Mastroyiannopoulos NP, Furling D, Uney JB, Phylactou LA Expression of miR-1, miR-133a, miR-133b and miR-206 increases during development of human skeletal muscle. BMC Dev Biol. 2011;11(1):34.

28. Kim HK, Lee YS, Sivaprasad U, Malhotra A, Dutta A. Muscle-specific microRNA miR-206 promotes muscle differentiation. J Cell Biol. 2006;174(5):677-87.

29. Lin S, Li H, Mu H, Luo W, Li Y, Jia X, et al. Let-7b regulates the expression of the growth hormone receptor gene in deletion-type dwarf chickens. BMC Genomics. 2012;13(1):306.

30. Ørom UA, Nielsen FC, Lund AH. MicroRNA-10a binds the $5^{\prime}$ UTR of ribosomal protein mRNAs and enhances their translation. Mol Cell. 2008:30(4):460-71

31. Wang S, Aurora AB, Johnson BA, Qi X, McAnally J, Hill JA, et al. The endothelial-specific microRNA miR-126 governs vascular integrity and angiogenesis. Dev Cell. 2008;15(2):261-71. 
32. Rivas DA, Lessard SJ, Rice NP, Lustgarten MS, So K, Goodyear LJ, et al. Diminished skeletal muscle microRNA expression with aging is associated with attenuated muscle plasticity and inhibition of IGF-1 signaling. FASEB J. 2014;28(9):4133-47.

33. Goettsch C, Rauner M, Pacyna N, Hempel U, Bornstein SR, Hofbauer LC. miR-125b regulates calcification of vascular smooth muscle cells. Am J Pathol. 2011;179(4):1594-600.

34. Cardinali B, Castellani L, Fasanaro P, Basso A, Alema S, Martelli F, et al. Microrna-221 and microrna-222 modulate differentiation and maturation of skeletal muscle cells. PLoS One. 2009;4(10):e7607.

35. Babiychuk EB, Palstra RJ, Schaller J, Kampfer U, Draeger A. Annexin VI participates in the formation of a reversible, membrane-cytoskeleton complex in smooth muscle cells. J Biol Chem. 1999;274(49):35191-5.

36. Chen L, Li YS, Cui J, Ning JN, Wang GS, Qian GS, et al. MiR-206 controls the phenotypic modulation of pulmonary arterial smooth muscle cells induced by serum from rats with hepatopulmonary syndrome by regulating the target gene, annexin A2. Cell Physiol Biochem. 2014;34(5):1768-79.

37. Lawlor MW, DeChene ET, Roumm E, Geggel AS, Moghadaszadeh B, Beggs AH. Mutations of tropomyosin 3 (TPM3) are common and associated with type 1 myofiber hypotrophy in congenital fiber type disproportion. Hum Mutat. 2010;31(2):176-83.

38. Shi Y, An J, Liang J, Hayes SE, Sandusky GE, Stramm LE, et al. Characterization of a mutant pancreatic elF-2alpha kinase, PEK, and co-localization with somatostatin in islet delta cells. J Biol Chem. 1999;274(9):5723-30.

39. De Mario A, Quintana-Cabrera R, Martinvalet D, Giacomello M. (Neuro) degenerated Mitochondria-ER contacts. Biochem Biophys Res Commun. 2017;483(4):1096-109.

40. Mutryn MF, Brannick EM, Fu W, Lee WR, Abasht B. Characterization of a novel chicken muscle disorder through differential gene expression and pathway analysis using RNA-sequencing. BMC Genomics. 2015;16(1):399.

41. Oberc MA, Engel WK. Ultrastructural localization of calcium in normal and abnormal skeletal muscle. Lab Investig. 1977;36(6):566-77.

42. Byrd SK. Alterations in the sarcoplasmic reticulum: a possible link to exercise-induced muscle damage. Med Sci Sports Exerc. 1992;24(5):531-6.

43. Emery AE, Burt D. Intracellular calcium and pathogenesis and antenatal diagnosis of Duchenne muscular dystrophy. Br Med J. 1980;280(6211):355-7.

44. Powder KE, Ku Y, Brugmann SA, Veile RA, Renaud NA, Helms JA, Lovett M. A cross-species analysis of microRNAs in the developing avian face. PLoS One. 2012:7(4):e35111.

45. Choi B, Ryu DY, Ryoo I-G, Kwak M. NFE2L2/NRF2 silencing-inducible miR-206 targets c-MET/EGFR and suppresses BCRP/ABCG2 in cancer cells. Oncotarget. 2017;8(63):107188.

46. Ramnarayanan S, Kyathanahalli C, Ingles J, Park-York M, Jeyasuria P, Condon $J C$. The unfolded protein response regulates uterine myocyte antioxidant responsiveness during pregnancy. Biol Reprod. 2016;95(6):120 1-9.

47. Simic T, Savic-Radojevic A, Pljesa-Ercegovac M, Matic M, Mimic-Oka J. Glutathione S-transferases in kidney and urinary bladder tumors. Nat Rev Urol. 2009;6(5):281.

48. Zhou N, Lee WR, Abasht B. Messenger RNA sequencing and pathway analysis provide novel insights into the biological basis of chickens' feed efficiency. BMC Genomics. 2015;16(1):195.

Ready to submit your research? Choose BMC and benefit from:

- fast, convenient online submission

- thorough peer review by experienced researchers in your field

- rapid publication on acceptance

- support for research data, including large and complex data types

- gold Open Access which fosters wider collaboration and increased citations

- maximum visibility for your research: over $100 \mathrm{M}$ website views per year

At BMC, research is always in progress.

Learn more biomedcentral.com/submissions 